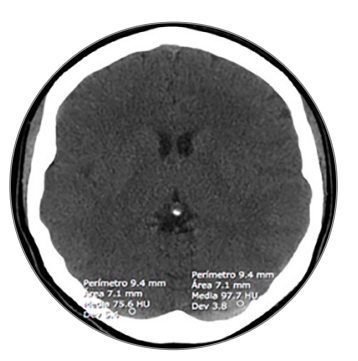

\title{
Densidad escanográfica de los senos venosos en pacientes sanos a 2.600 metros sobre el nivel del mar
}

\author{
Computed Tomography Density of the Venous Sinuses in Healthy \\ Patients at 2,600 Meters Above Sea Level
}

Santiago Andrade Sofía Catalina Velasco Aníbal J. Morillo' Sonia Bermúdez

Gustavo A. Triana'

\section{Palabras clave (DeCS)}

Hematocrito

Trombosis de los senos intracraneales

Tomografía

computarizada

multidetector

Imagen por resonancia

magnética

\section{Key words (MeSH)}

\section{Hematocrit}

Sinus thrombosis,

intracranial

Multidetector computed tomography

Magnetic resonance imaging
'Radiólogo(a) Departamento de Radiología e Imágenes Diagnósticas, Fundación Santa Fe de Bogotá. Universidad El Bosque. Bogotá, Colombia.

\section{Resumen}

Introducción: Existe relación entre la densidad escanográfica de las estructuras vasculares medida en unidades Hounsfield (UH) y el hematocrito, el cual se incrementa en las personas que viven a mayor altitud sobre el nivel del mar. Se ha descrito que una densidad de $70 \mathrm{UH}$ es el valor límite superior de normalidad de densidad de los senos venosos en personas sin trombosis venosa que habitan a una altitud de $1.000 \mathrm{~m} \mathrm{s.} \mathrm{n}$. m.; sin embargo, no se ha establecido esta medida en personas que habitan en altitudes geográficas mayores. En este estudio se determinó dicho valor para personas sin trombosis de senos venosos que viven a 2.600 m s. n. m. Objetivo: Este estudio tiene como propósito caracterizar la densidad normal de los senos venosos en pacientes que habitan en altitudes geográficas de $2.600 \mathrm{~m} \mathrm{s.} \mathrm{n}$. $\mathrm{m}$. Metodología: Se analizaron las densidades escanográficas de los senos venosos longitudinal superior y de los sitios de unión entre los senos transversos y senos sigmoides de 240 sujetos que habitan a 2.600 m s. n. m., quienes asistieron a la institución entre enero de 2008 y junio de 2016. Estas personas consultaron por cefalea, convulsiones, alteración del estado de conciencia o focalización neurológica. A estos pacientes se les realizó inicialmente un estudio escanográfico de cráneo simple, hemoglobina y hematocrito con diferencia no mayor a 3 días entre el estudio escanográfico y el hemograma, así como estudios confirmatorios de positividad o ausencia de trombosis de senos intracraneales mediante resonancia magnética simple o angiorresonancia. Resultados: La media de UH fue de 59,8 con un rango entre 43,2 y 74,9, para pacientes normales. Se encontró una correlación positiva de 0,49 entre el hematocrito y las UH como es ampliamente conocido en la literatura. Conclusión: El rango en UH de los pacientes sin trombosis de senos venosos es amplio y supera por 4 puntos el informado en la literatura. Esto puede ser atribuible al aumento del hematocrito en individuos que viven a 2.600 m s. n. m. Para establecer normalidad en los pacientes con valores superiores a $70 \mathrm{UH}$ se sugiere tomar en cuenta la simetría de la densidad escanográfica con respecto al seno venoso contralateral, así como evaluar la morfología del borde anterior del seno venoso y la adecuada localización del ROI (Region of Interest) para medir la densidad venosa.

\section{Summary}

Introduction: There is a relationship between the intravascular density measured in Hounsfield units (HU) in computed tomography (CT) and the hematocrit levels, which increases in people who live above sea level. The expected density in venous sinuses is lower than $70 \mathrm{HU}$ in healthy people living at 1000 meters above sea level. However, this measure has not been established in people living at higher geographic altitudes. In this study, this value was determined for normal people living at 2,600 meters above sea level. Objective: The purpose of this study is to characterize the normal density of the venous sinuses in patients who live at geographical altitudes of 2,600 m above sea level. Methodology: We measured the density on CT at the superior longitudinal venous sinus and at the junction between the transverse sinus and sigmoid sinus of 240 subjects living at 2600 meters above sea level from January 2008 to June 2016. These patients consulted for headache, seizures, altered state 
of consciousness or neurological focus. Initially, all participants had a non-enhanced CT brain, hemoglobin and hematocrit levels with a difference of no more than 3 days between the CT study and the hemogram, as well as confirmatory studies of positivity or absence of intracranial sinus thrombosis by non-enhanced MR or MRA. Results: The mean HU was 59.8 with a range between 43.2 and 74.9, for normal patients. A positive correlation of 0.49 was found between hematocrit and HU, as is widely known in the literature. Conclusion: We obtained a wide range in the HU of healthy patients compared to the values reported in other papers, and exceeds the highest value by 4 points. This may be attributable to the increased hematocrit in healthy patients living at 2,600 meters above sea level. In order to establish whether patients with venous sinus densities greater than $70 \mathrm{HU}$ have venous thrombosis, our suggestion is to evaluate the symmetry of the density compared to the contralateral venous sinus, as well as to evaluate the morphology and the location of the ROI used to measure venous density.

\section{Introducción}

En la actualidad, los programas de visualización de imágenes diagnósticas en formato digital permiten la medición de la densidad de las diferentes estructuras corporales en la modalidad de escanografía, también llamada tomografía computarizada (TC). Esta densidad se expresa globalmente en unidades Hounsfield (UH) y se obtiene automáticamente por el programa de visualización de imágenes. La medición se obtiene al seleccionar un área de interés (ROI, por sus siglas en inglés Region of interest) que debe abarcar aproximadamente el $75 \%$ del área de la estructura por medir.

A pesar de la amplia aceptación de la relación entre la densidad escanográfica y el hematocrito, no se han definido valores límite de normalidad de densidad de los senos venosos intracraneales en personas normales para diferenciarlos de los pacientes con trombosis de senos venosos en altitudes geográficas mayores a 1.000 m. s. n. m.. La sospecha de trombosis de senos venosos se basa en apreciaciones subjetivas al visualizar una mayor densidad de la habitual en estas estructuras vasculares mientras se evalúa un estudio escanográfico. En la institución donde se hizo el presente estudio, ubicada en una altitud geográfica de 2.600 m. s. m. n., se ha observado frecuente variabilidad interobservador, así como subdiagnóstico de esta patología. Comúnmente se atribuye la alta densidad de las estructuras vasculares intracraneales a valores elevados de hematocrito.

Para que la evaluación de los estudios escanográficos en pacientes con sospecha de trombosis de senos venosos sea objetiva, se debe realizar la medición cuantitativa de la densidad de los senos venosos en UH. Existe una correlación positiva $(r=0,840)$ entre la densidad vascular medida en UH y el valor de hematocrito (1). Se conoce que a mayor altitud geográfica, aumenta la concentración eritrocitaria intravascular (hematocrito); por lo tanto, los valores de densidad vascular son mayores a mayor altitud geográfica.

A mayor altitud s. n. m., es menor la concentración ambiental de oxígeno; como mecanismo corporal compensatorio, las personas que habitan o ascienden a altitudes geográficas superiores a 2.500 m. s. n. $\mathrm{m}$., aumentan su concentración eritrocitaria por mayor producción de eritropoyetina (2), lo cual incrementa los niveles de hematocrito. En consecuencia, se puede observar mayor densidad vascular $(3,4)$.

El hematocrito se define como el porcentaje de masa eritrocitaria en $100 \mathrm{~mL}$ de sangre. Los rangos de normalidad en mujeres y hombres adultos en Bogotá, ubicada aproximadamente a 2.600 m. s. n. m., están entre 43-50 \% y 45-53\%, respectivamente (5). En los últimos 15 años, en estudios realizados con el propósito de identificar estados de anemia mediante imágenes, se demostró correlación entre los niveles de hemoglobina y la densidad vascular en distintas estructuras corporales $(6,7)$. La densidad vascular se debe cuantificar en escanografía simple, debido a que los medios de contraste yodados endovenosos aumentan de manera importante los valores de esta densidad .

Se ha informado $70 \mathrm{UH}$ como el valor máximo de normalidad de densidad en estructuras vasculares en personas que habitan a nivel del mar. Los valores superiores hacen sospechar trombosis de senos venosos intracraneales (8). Ninguno de los pacientes sin trombosis de senos venosos en dicho estudio mostró valores de densidad superiores a 70 UH. Para la atención de personas que habitan en lugares a mayor altitud sobre el nivel de mar, se considera importante realizar un estudio para establecer los valores de densidad vascular en pacientes sin trombosis de senos venosos.

Realizar una medición objetiva de la densidad de los senos venosos y caracterizar esta población por el aumento en el hematocrito $-\mathrm{y}$, por tanto, en la densidad - permitirá alertar sobre la aparición de entidades, como trombosis de senos venosos, con menores consecuencias neurológicas a largo plazo.

\section{Métodos}

Después de la aprobación del comité de ética de las instituciones involucradas se realizó la revisión de las historias clínicas e imágenes de escanografía, resonancia magnética simple y angiorresonancia de 243 paciente, entre 2008 y 2016, sin trombosis de senos venosos confirmado por resonancia magnética cerebral como en la figuras 1 y 2. En el proceso de análisis se identificaron y se excluyeron del estudio tres pacientes sin trombosis de senos venosos, en quienes no se encontraron suficientes datos clínicos, y uno que no cumplía los factores técnicos para la medición.

Se realizó también la medición de senos venosos de pacientes con diagnóstico de trombosis de senos venosos que tenían estudio confirmatorio de resonancia magnética (figuras 3,4 y 5).

\subsection{Población}

Los pacientes eran mayores de 18 años con escanografía cerebral simple normal, a quienes además se les realizó RM y cuadro hemático completo, con una diferencia de tiempo entre los estudios de laboratorio no mayor a 3 días con respecto a la escanografía.

Se excluyeron los pacientes con cualquier dispositivo intracraneal o lesiones que produjeran artefacto o alteración de la densidad de los senos venosos. También, pacientes con hemorragia intracraneana, antecedente de trauma reciente y patología intracraneal diferente a trombosis de senos venosos que produjera alteración morfológica en la escanografía. Por último, no se incluyeron pacientes a quienes se les hubiera administrado medio de contraste endovenoso durante los 3 días previos al estudio de escanografía cerebral simple. 

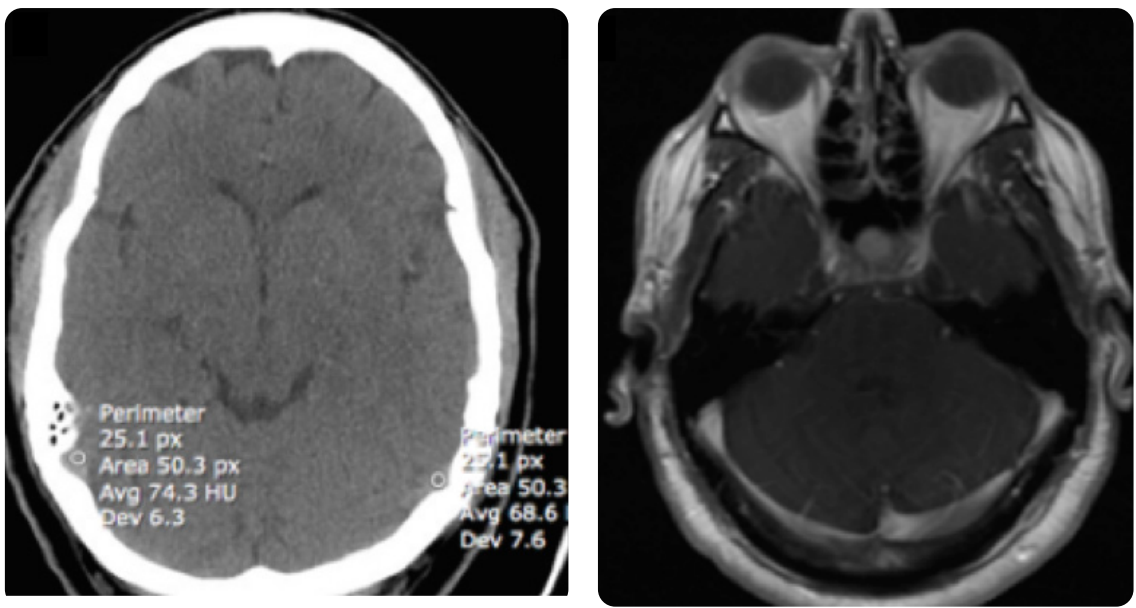

Figura 1. Hombre de 49 años con cefalea, Hb: 19,1 g/ dL, Hto: 59. a) Corte axial de escanografía sin medio de contraste. Aumento de la densidad venosa con valores superiores a $65 \mathrm{UH}$ en el seno transverso izquierdo y a $70 \mathrm{UH}$ en el derecho. b) Imagen axial de RM secuencia eco de espín con información T1 con medio de contraste. Se evidencia permeabilidad de senos transversos, lo que confirma que no hay trombosis.
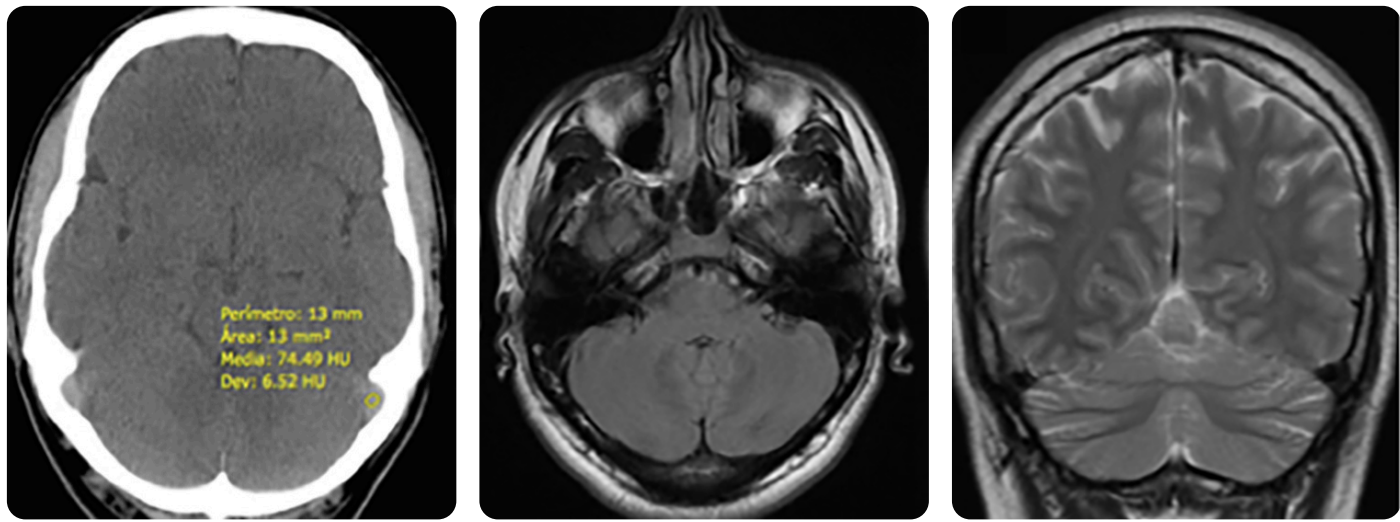

Figura 2. Hombre de 32 años con cefalea, $\mathrm{Hb}: 17,3 \mathrm{~g} / \mathrm{dL}$ Hto: 50,3. a) Corte axial de escanografía sin medio de contraste. Aumento de la densidad venosa en seno transverso izquierdo con densidad de $74 \mathrm{UH}$ en esta localización. b y c) Imagen axial y coronal de RM, secuencias eco de espín con información $\mathrm{T} 1$ y $\mathrm{T} 2$, respectivamente, sin alteración en el signo vacío de flujo ni en la morfología de los contornos de los senos venosos.

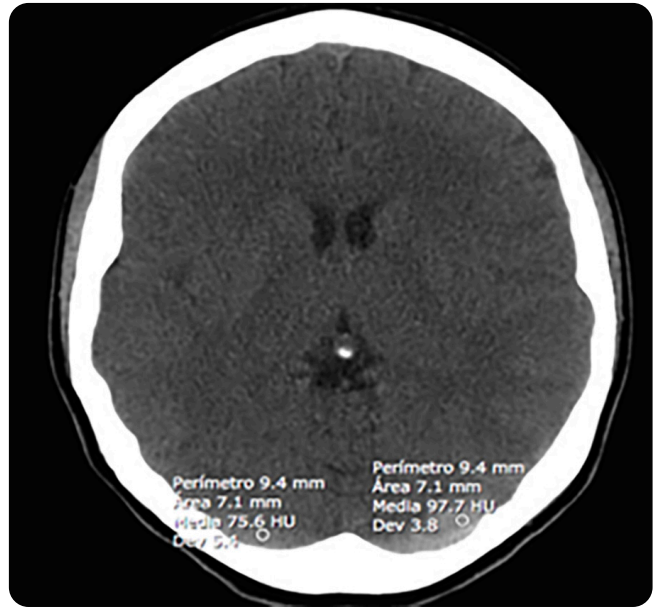

Figura 3. Mujer de 21 años con cefalea y vértigo. Corte axial de escanografía sin medio de contraste. Se observa asimetría en la densidad de los senos transversos e importante aumento en la densidad del seno transverso izquierdo con valor indicativo de trombosis.

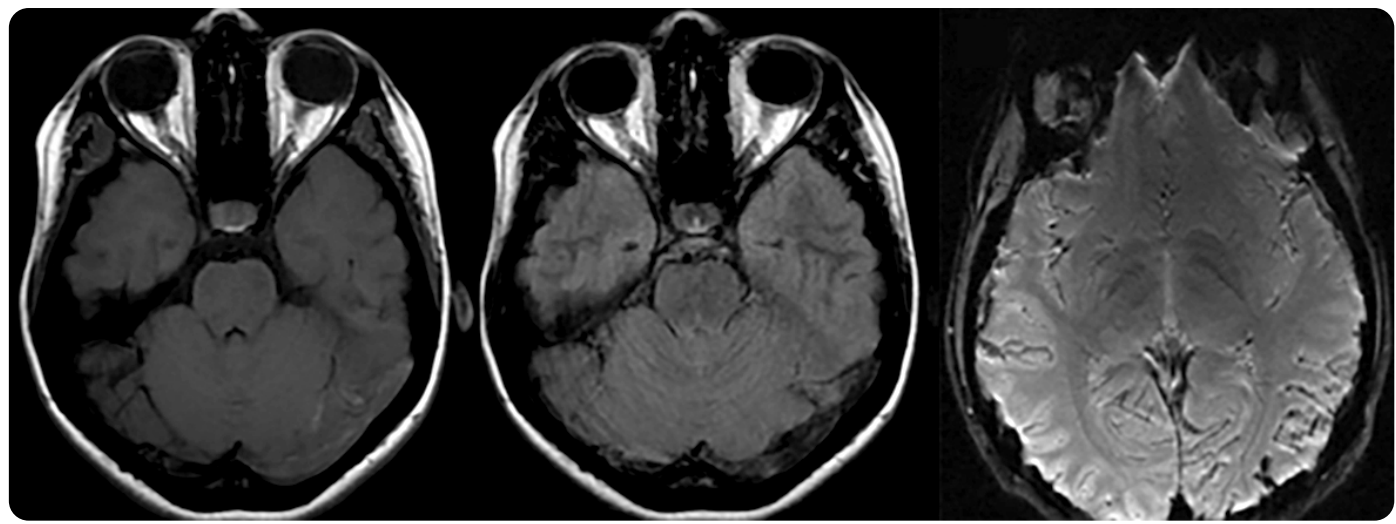

Figura 4. Misma paciente de estudio escanográfico previo. Estudio de RM cerebral. a) Imagen axial, secuencia eco de espín con información T1: prominencia y aumento en la intensidad de señal del seno transverso izquierdo por trombosis. b) Axial T2 FLAIR: alteración del signo de vacío de flujo del seno transverso izquierdo, configuración convexa del contorno anterior. c) Axial Susceptibilidad Magnética. Baja señal de seno transverso izquierdo, seno longitudina superior y algunas venas corticales occipitales por trombosis. 

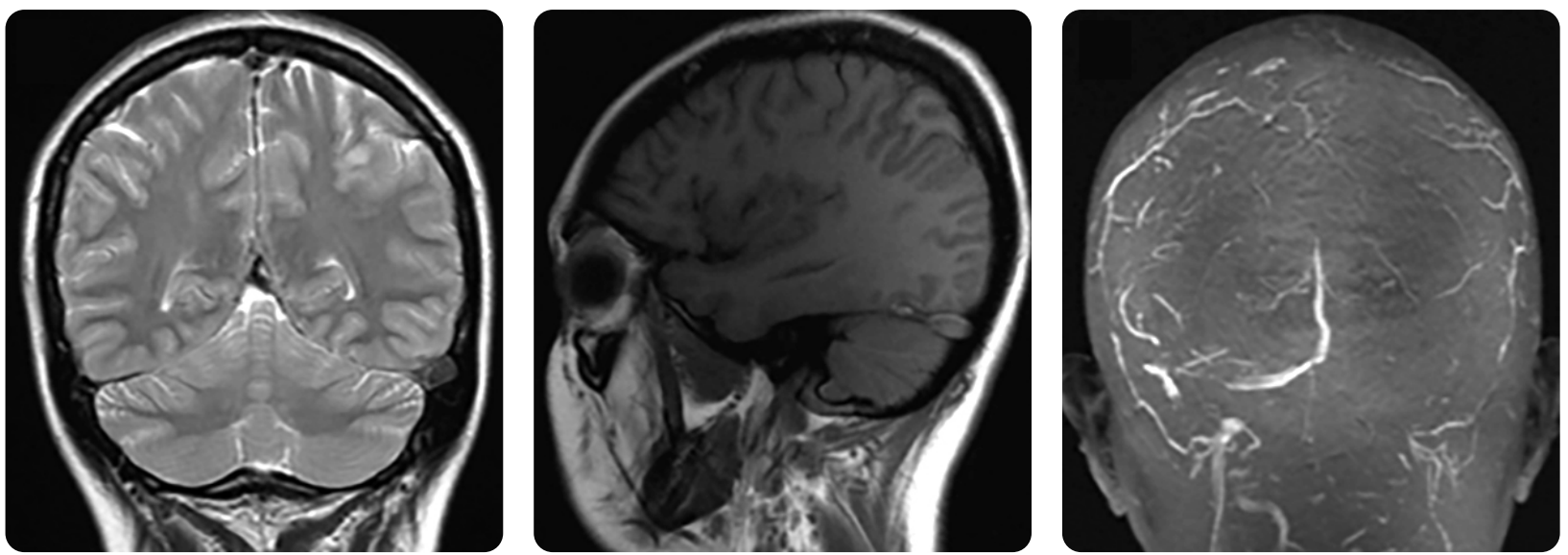

Figura 5. Misma paciente de estudio escanográfico previo. Estudio cerebral y venografía por RM. a) Coronal secuencia eco de espín con información T2. Prominencia y heterogeneidad en la intensidad de señal de los senos longitudinal superior y transverso izquierdo por trombosis, contornos convexos de los diferentes bordes de la estructura vascular. b) Sagital T1 Flair. Aumento en la intensidad de señal del seno transverso izquierdo y contorno anterior y superior convexo. c) Venografía por RM. Ausencia de flujo vascular en un extenso segmento del seno longitudinal superior, así como en la totalidad del seno venoso transverso izquierdo y sigmoide.

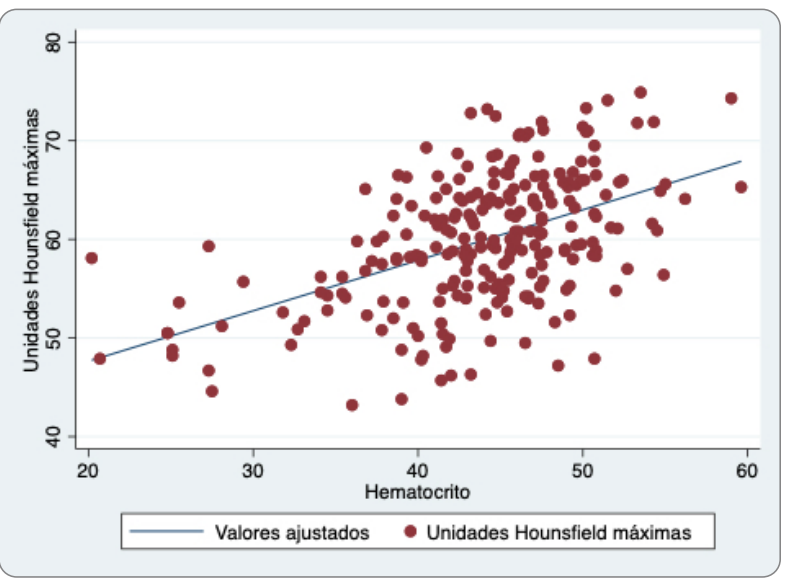

Figura 6. Relación entre densidad escanográfica $(\mathrm{UH})$ y hematocrito de la población estudiada sin trombosis de senos venosos, muestra correlación positiva de 0,49. Se observan, además, los valores de densidad escanográfica mayores a $70 \mathrm{UH}$, con un valor máximo de 74,9 UH.

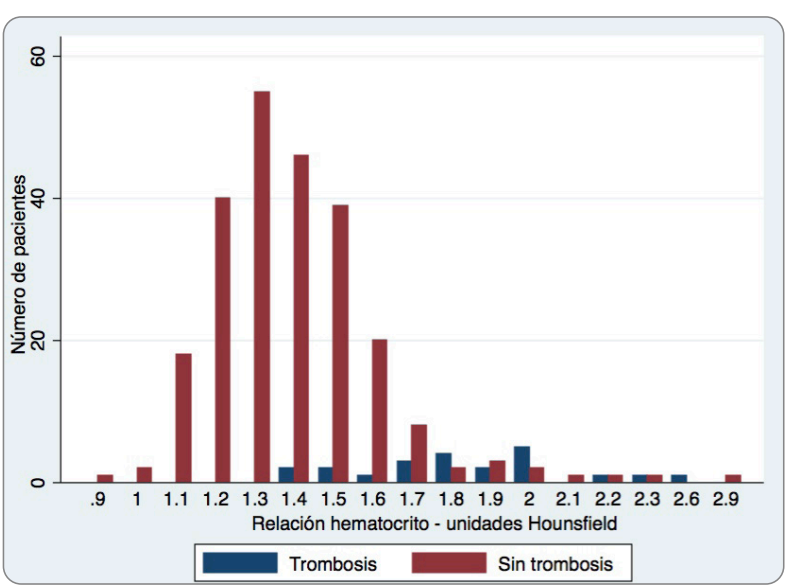

Figura 7. Número de pacientes con valores de relación $\mathrm{H}: \mathrm{H}$ tanto en el grupo de pacientes con trombosis de senos venosos (azul) como en el grupo sin trombosis de senos venosos (rojo). El valor límite a partir del cual se presentaron casos de trombosis fue 1,4.

\subsection{Tamaño de muestra}

Con el fin de establecer un límite de normalidad superior para personas que habitan a $2.600 \mathrm{~m}$. s. n. m., se realizaron las mediciones de densidad en 240 pacientes desde 2008 hasta 2016 en estudios de TAC cerebral simple, que cumplían los criterios de inclusión. Este tamaño de muestra supera la mayor muestra informada en la literatura, que es de 166 pacientes (1). Nótese que en este estudio se realizó un muestreo consecutivo dado que se seleccionaron todos los pacientes disponibles con diagnóstico de trombosis de senos venosos con TAC y RM, y hematocrito.

\section{Materiales}

Las escanografías incluidas en el estudio se realizaron en un tomógrafo General Electric Light Vision VCT y las imágenes fueron adquiridas usando los siguientes parámetros técnicos: 120 kilovoltios, 300 miliamperios, espesor de corte de $5 \mathrm{~mm}$, tiempo de rotación de 1 , 20 detectores y FOV (campo de visión) de $240 \mathrm{~mm}$.

La medición de densidad de los senos venosos en los pacientes sin trombosis se realizó en tres sitios: la confluencia de los senos, el tercio inferior del seno longitudinal superior y en las uniones transversosigmoideas derecha e izquierda. Para dicha medición se utilizó un ROI con áreas entre 6 y $15 \mathrm{~mm}^{2}$ ocupando entre el 40 y el $75 \%$ del calibre del seno a medir, como se muestra en la figura 3 .

\subsection{Análisis estadístico}

Se utilizaron medidas estadísticas de tendencia central para describir y graficar los datos. Se utilizó la prueba de Shapiro-Wilk para determinar si la muestra sigue una distribución normal. Igualmente, se realizó una prueba de correlación entre el hematocrito y la densidad vascular medida en UH. El análisis estadístico se hizo utilizando STATA 12.

\section{Resultados}

Se estudiaron un total de 240 pacientes sin trombosis de senos venosos, con una media de edad de 47 años (rango de 18 a 97 años), la mayoría eran hombres, 122 sujetos $(51 \%)$. La media de hemoglobina en esta población fue de 14,59 (rango 6,6-19.1), el hematocrito tuvo una 
media de 43,86 con un rango de 20,2 a 59,6 (tablas 1 y 2). Se evaluó, además, la densidad escanográfica en senos venosos de 22 pacientes con trombosis confirmada (tabla 3 ).

A continuación, se muestran los resultados de los rangos, media y mediana de la densidad venosa en $\mathrm{UH}$, hemoglobina, hematocrito, y la relación UH:Hto.

Tabla 1. Características de individuos sin trombosis de senos venosos tomados aleatoriamente como grupo control

\begin{tabular}{|l|l|l|l|l|}
\hline Características & \multicolumn{1}{c|}{ Media } & Mediana & \multicolumn{1}{c|}{ Min } & \multicolumn{1}{c|}{ Max } \\
\hline Edad & 46,63768 & & 18 & 82 \\
\hline UH & 60,34464 & 59,85 & 46,3 & 74,6 \\
\hline Hemoglobina & 14,72754 & 14,9 & 7,1 & 18,7 \\
\hline Hematocrito & 44,41159 & 44,7 & 20,7 & 56,2 \\
\hline H:H & 1,381159 & 1,4 & 1,1 & 2,3 \\
\hline
\end{tabular}

Nota: $\mathrm{H}: \mathrm{H}=$ Relación UH/Hto.

Tabla 2. Características de mujeres y hombres sin trombosis de senos venosos

\begin{tabular}{|c|c|c|c|c|}
\hline \multicolumn{5}{|c|}{ Mujeres } \\
\hline Características & Media & Mediana & Min & Max \\
\hline Edad & 45,82 & 42,5 & 18 & 92 \\
\hline UH & 58,47 & 59,05 & 43,8 & 71,8 \\
\hline Hemoglobina & 13,67 & 14,1 & 6,6 & 18,1 \\
\hline Hematocrito & 41,19 & 42,2 & 20,2 & 53,3 \\
\hline H:H & 1,44 & 1,4 & 1,1 & 2,9 \\
\hline \multicolumn{2}{|c|}{} & Hombres & & \\
\hline Edad & 48,72 & 49,5 & 18 & 97 \\
\hline UH & 61,19 & 61,25 & 43,2 & 74,9 \\
\hline Hemoglobina & 15,48 & 15,8 & 7,9 & 19,1 \\
\hline Hematocrito & 46,44 & 47,3 & 24,8 & 59,6 \\
\hline H:H & 1,33 & 1,3 & 0,9 & 2 \\
\hline
\end{tabular}

Tabla 3. Características del total de pacientes con trombosis de senos venosos

\begin{tabular}{|c|c|c|c|c|}
\hline Características & Media & Mediana & Min & Max \\
\hline Edad & 40,86 & & 19 & 94 \\
\hline UH & 75,5 & 76,9 & 69,2 & 97,6 \\
\hline Hemoglobina & 14,5 & 43,9 & 10,1 & 17,2 \\
\hline Hematocrito & 43,7 & 14,4 & 31,1 & 53,5 \\
\hline H:H & 1,8 & 1,7 & 1,4 & 2,6 \\
\hline
\end{tabular}

En la tabla 4 se muestran los valores de densidad máximos y mínimos, así como la media de los senos venosos transversos y de la confluencia de los senos por separado en hombres y mujeres sin trombosis de senos venosos.
Tabla 4. Densidad de senos venosos medida en unidades Hounsfield en pacientes sin trombosis venosa según localización

\begin{tabular}{|c|c|c|c|c|}
\hline \multicolumn{5}{|c|}{ Mujeres } \\
\hline Características & Media & Mediana & Min & Max \\
\hline Seno transverso derecho & 57,11 & 56,5 & 38 & 71,8 \\
\hline Seno transverso izquierdo & 56,17 & 57,8 & 40,7 & 74,1 \\
\hline Confluencia de los senos & 51,08 & 43,9 & 32,8 & 67,5 \\
\hline \multicolumn{7}{|c|}{ Hombres } \\
\hline Seno transverso derecho & 58,89 & 60 & 31,2 & 74,9 \\
\hline Seno transverso izquierdo & 59,36 & 59,4 & 31,4 & 74,1 \\
\hline Confluencia de los senos & 52,95 & 53,05 & 36,6 & 71,3 \\
\hline
\end{tabular}

Se estableció una correlación positiva de 0,49 entre el Hto. y las $\mathrm{UH}$ en pacientes sin trombosis de senos venosos, como se observa en la figura 6. La media de la relación UH-Hto. es de 1,4 (rango 0,9-2,9).

La media de UH máximas fue de 59,8 con un rango de 43,2 a 74,9 (Shapiro-Wilk $p$ 0,61549, se falla en rechazar la hipótesis nula de normalidad). Adicionalmente, se obtiene una correlación positiva entre el hematocrito y las UH de 0,5 , como se observa en la figura 6 .

El valor umbral de corte de 1,8 en la relación $\mathrm{H}: \mathrm{H}$ (UH:Hto) sugerido por Black et al. (8) y de 1,5 sugerido por Buick et al. (9), no coincidió con los hallazgos en nuestra población, la cual sí presentó sobreposición en la distribución de los valores de la relación (figura 7), documentándose valores de relación $\mathrm{H}: \mathrm{H}$ de hasta 1,4 en pacientes con trombosis (tabla 5), ya que al presentar mayores niveles de hematocrito el valor de la relación $\mathrm{H}: \mathrm{H}$ disminuye.

Tabla 5. Relación H:H

\begin{tabular}{|c|c|c|c|c|}
\hline \multicolumn{5}{|c|}{ Hombres } \\
\hline Características & Media & Mediana & Min & Max \\
\hline $\mathrm{H}: \mathrm{H}$ & 1,33 & 1,3 & 0,9 & 2 \\
\hline $\mathrm{H}: \mathrm{H}$ & Mujeres \\
\hline
\end{tabular}

\section{Discusión}

En la literatura se ha documentado ampliamente la correlación positiva entre el hematocrito y la densidad vascular medida en UH. Así mismo, se ha establecido una relación directamente proporcional entre la altitud geográfica y el aumento del hematocrito (1-3,8,9). Por lo tanto, se espera que a mayor altura se encuentren mayores valores de densidad vascular medida en UH.

Comparativamente, mientras en el estudio de Black et al.(8) ninguno de los controles tenía valores superiores a $70 \mathrm{UH}$, en el presente trabajo se encontraron 18 pacientes sin trombosis de senos venosos con densidad vascular mayor a $70 \mathrm{UH}$, en una población sana distribuida normalmente; se obtuvo un valor máximo de 74,9 UH.

Nótese que la densidad en el estudio mencionado se midió en la confluencia de los senos venosos, igual que en nuestro estudio. Con la salvedad de que en nuestra investigación se midió además la unión transversosigmoidea. Estas poblaciones no son comparables debido a 
que la altura en Bogotá es mayor que la de la ciudad donde se realizó el estudio de Black et al.

Se debe mencionar que 9 de los 18 controles sanos con densidad mayor a $70 \mathrm{UH}$, tenían valores de hemoglobina y hematocrito superiores a los esperados - únicamente por habitar a gran altitud - y fueron considerados casos de poliglobulia (niveles de hemoglobina $>16 \mathrm{~g} / \mathrm{dL}$ en mujeres y $>16,5 \mathrm{~g} / \mathrm{dL}$ en hombres o hematocrito y hematocrito $>48 \%$ en mujeres y $>49 \%$ en hombres) (10); por lo expuesto, la poliglobulia se debe considerar como diagnóstico diferencial en el hallazgo de alta densidad de los senos venosos, especialmente en altitudes iguales o superiores a 2.600 m. s. n. m., donde, como respuesta fisiológica a la menor cantidad de oxígeno, los niveles de hemoglobina y hematocrito son mayores. Por otro lado, del total de la población, 43 tenían criterios de poliglobulia y no se encontraron valores de densidad de los senos venosos mayores a $70 \mathrm{UH}$.

En este estudio no se tuvo en cuenta el tiempo entre el inicio de los síntomas y el estudio escanográfico; por lo tanto, no fue posible definir la relación entre la densidad y la cronicidad del evento trombótico. El tiempo de evolución del evento trombótico altera la densidad del trombo, siendo mayor su densidad durante la fase aguda de la trombosis y disminuyendo de forma progresiva en fases subaguda y crónica. La recanalización parcial del vaso también disminuiría su densidad, por lo tanto los valores bajos de densidad escanográfica no descartan eventos trombóticos no agudos o con recanalización. La presentación del trombo en RM es variable debido a los cambios en el estado de degradación de la hemoglobina; en las secuencias eco de espín puede mostrar ausencia del signo de vacío de señal, con intensidad media en T1 y baja señal en T2 en fase aguda, mientras que en la fase subaguda se observa alta señal en T1. La secuencia eco de gradiente con información T1 con medio de contraste brinda la mayor sensibilidad y especificidad para la detección del trombo.

Otros grupos de investigación, como Alsafi et al. (11), han comparado la relación entre la densidad de senos venosos trombosados con el promedio de densidad de las arterias carótidas internas intracraneales y han referido un relación mayor a 1,5 como altamente sugestiva de trombosis de senos venosos; sin embargo, la finalidad de nuestro estudio se enfocó exclusivamente en el análisis de la densidad de los senos venosos, ya que las relaciones con otras estructuras vasculares implica mayor tiempo en la aproximación diagnóstica. El frecuente compromiso ateromatoso calcificado puede alterar los valores de densidad arterial $\mathrm{y}$, en consecuencia, la relación de la densidad del seno trombosado con el promedio de densidad arterial en carótidas internas supraclinoideas y la arteria basilar.

\section{Conclusión}

Nuevamente se demuestra correlación positiva entre la densidad escanográfica y el hematocrito, con valores de hasta 74,9 UH en pacientes sin trombosis de senos venosos, la cual es $5 \mathrm{UH}$ mayor a lo documentado en estudios previos en poblaciones que habitan a menor altitud geográfica. El valor mínimo de densidad en pacientes con trombosis de senos venosos fue de 69,2 UH; sin embargo, en este estudio no se definió el tiempo del evento trombótico.

Por los resultados obtenidos en el presente estudio se considera apropiado que en pacientes con valores entre 69 y 74,9 UH que habiten a altitudes geográficas similares a la de Bogotá, se evalúen otras características escanográficas que sugieran trombosis de senos venosos, como la configuración convexa en los contornos anterior y superior de los senos transversos especialmente en plano sagital; también se considera útil evaluar la asimetría en la densidad y el calibre de los senos venosos, siempre y cuando se excluya hipoplasia del seno venoso contralateral y otras variantes anatómicas (12).

Debe tenerse en cuenta que existen diversos factores técnicos que pueden alterar la medición de la densidad, dentro de estos, artificios por endurecimiento del haz secundario a la presencia de dispositivos intra o extracraneales, así como variaciones de un escanógrafo a otro (13).

\section{Referencias}

1. Lee SY, Cha S-H, Lee S-H, Shin D-I. Evaluation of the effect of hemoglobin or hematocrit level on dural sinus density using unhanced computed tomography. Yonsei Med J. 2013;54(1):1-10.

2. Faura J, Ramos J, Reynafarje C, English E, Finne P, Finch C. Effect of altitude on erytropoiesis. Blood J. 1969;33(5):668-76.

3. Huff R, Lawrence J, Siri W, Wasswerman L, Hennessy T. Effects of changes in altitude on hematopoietic activity. Medicine. 1951;30(3):197-217.

4. New PF, Aronow S. Attenuation measurements of whole blood and blood fractions in computed tomography. Radiology. 1976;121(September):635-40.

5. Benavides W. Ubicación altitudinal del umbral hipóxico para la masa total de hemoglobina en poblaciones colombianas. Bogotá: Universidad Nacional de Colombia; 2013.

6. Bruni SG, Patafio FM, Dufton Ja, Nolan RL, Islam O. The assessment of anemia from attenuation values of cranial venous drainage on unenhanced computed tomography of the head. Can Assoc Radiol J. 2013;64(1):46-50.

7. Collins AJ, Gillespie S, Kelly BE. Can computed tomography identify patients with anaemia? Ulster Med J. 2001;70(2):116-8.

8. Black DF, Rad E, Gray L, Campeau NG, Kallmes DF. Cerebral venous sinus density on noncontrast CT correlates with hematocrit. Am J Neuroradiol. 2011;32:1354-7.

9. Buyck PJ, De Keyzer F, Vanneste D, Wilms G, Thijs V, Demaerel P. CT density measurement and $\mathrm{H}: \mathrm{H}$ ratio are useful in diagnosing acute cerebral venous sinus thrombosis. Am J Neuroradiol. 2013;34(8):1568-72.

10. Tefferi A, Barbui T. Polycythemia vera and essential thrombocythemia: 2017 update on diagnosis, risk-stratification, and management. Am J Hematol. 2017;92:95-108.

11. Alsafi A, Lakhani A, Carlton-Jones L, Lobotesis K. Cerebral venous sinus thrombosis, a nonenchanced CT diagnosis. Radiol Res Pract. 2015, article ID 581437.

12. Canedo-Antelo M, Baleato-González S, Mosqueira AJ, Casas-Martínez J, Oleaga L, Vilanova JC, et al. Radiologic clues to cerebral venous thrombosis. RadioGraphics. 2019;39(6):1611-28.

13. Lamba R, McGahan JP, Corwin MT, Chin-Shang L, Tran T, Seibert JA, et al. CT Hounsfield numbers of soft tissues on unenhanced abdominal CT scans: variability between two different manufacturers' MDCT scanners. Am J Roentgenol. 2014;203:1013-20.

\section{Correspondencia}

Santiago Andrade Pérez

Calle 19 \# 14-95 casa 3

Ibagué, Colombia

dr.andradeperez@gmail.com

Recibido para evaluación: 28 de diciembre de 2020

Aceptado para publicación: 17 de febrero de 2021 